Mercator, Fortaleza, v. 16, e16002, 2017.

DOI: https://doi.org/10.4215/rm2017.e16002

ISSN: $1984-2201$

Copyright @ 2002, Universidade Federal do Ceará

\title{
NOUVEAUX PAYSAGES URBAINS DU PROGRAMME MINHA CASA, MINHA VIDA
}

THERY, H. ${ }^{\text {* }}$

(a) Directeur de recherche émérite au Centre de Recherche Et de Documentation sur les Amériques (CNRS-Creda). http://lattes.cnpq. $\mathrm{br} / 7931074433827497$

\section{(*) CORRESPONDING AUTHOR}

Address: Université Sorbonne Nouvelle-Paris 3. CREDA, 28, rue Saint Guillaume. 75007, Paris, France. Tel: 0144398671 Email: hthery@aol.com

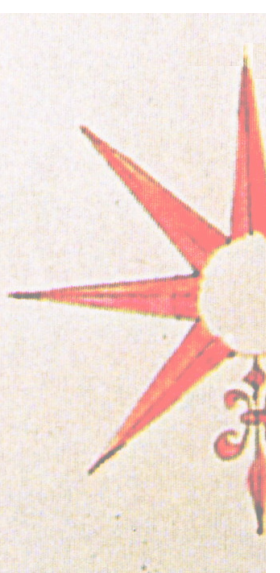

\section{RESUME}

Le programme "Minha casa, minha vida" (Ma Maison, Ma Vie), le plus grand programme de construction de logements des trente dernières années, a profondément changé le paysage de nombreuses villes brésiliennes en y créant d'immense quartiers de maisons identiques, alignées au cordeau et très proches les unes des autres. Lancé en 2009, au cours du second mandat de Luíz Inácio Lula da Silva, il est devenu l'un des principaux fleurons des politiques gouvernementales de Dilma Rousseff. Conçu pour stimuler la construction et lutter contre les effets de la crise économique internationale, il est devenu un instrument pour réduire le déficit de logements dans le pays, mais il n'échappe pas aux critiques des spécialistes qui l'ont étudié. L'article, après une brève présentation de son histoire, il cherche à repérer la marque du programme sur le paysage des villes sur des photos (prises sur le terrain ou récoltées sur Internet) et des images verticales de Google Earth. Des exemples spécifiques sont choisis dans des villes de taille moyenne et certaines capitales. Enfin deux interviews nous aident compléter l'évaluation du programme.

Mots cles: Politique de logement; Paysages urbains; Minha Casa Minha Vida.

\section{ABSTRACT/ RESUMO}

\section{NEW URBAN LANDSCAPES OF THE MINHA CASA, MINHA VIDA PROGRAM}

The "Minha casa, minha vida" (My house, My life) program, the country's largest housing program in the last 30 years, changed the landscape of many Brazilian cities. Launched in 2009, in the second term of former President Luiz Inacio Lula da Silva, he became one of Dilma Rousseff's main government policies Rousseff and was responsible for the construction, on the urban fringes around the country, of sets of tens, hundreds or even thousands small houses. Designed to streamline construction and combat the effects of the international economic crisis, it became an instrument to reduce the housing deficit in the country, but does not escape criticism from the experts who have studied their ventures. The article after a short presentation of its history, discusses its effects on the landscape of cities, measured through photos taken in field or collected on the Internet and Google Earth vertical images. Specific examples are chosen in medium-sized cities, and in some capitals. Finally two interviews help complete the program evaluation.

Keywords: Housing policies; Urban landscapes; Minha Casa Minha Vida.

\section{NOVAS PAISAGENS URBANAS DO PROGRAMA MINHA CASA, MINHA VIDA}

O programa "Minha casa, minha vida", maior programa habitacional do país nos últimos trinta anos, mudou profundamente a paisagem de muitas cidades brasileiras. Lancado em 2009, no segundo mandato do ex-presidente Lula, se tornou uma das principais bandeiras políticas do governo Dilma Rousseff e foi responsável da construção, nas periferias urbanas do país, de conjuntos de dezenas, centenas ou até milhares de pequenas casas. Concebido para dinamizar a construção civil e combater os efeitos da crise econômica internacional, se tornou um instrumento para reduzir o déficit habitacional do país, mas não escapa de críticas dos especialistas que estudaram seus empreendimentos. O presente artigo após ume breve apresentação da sua história, considera os seus efeitos na paisagem das cidades, medidos através de fotos, provenientes do campo ou da Internet, e de imagens verticais do Google Earth. Casos específicos são escolhidos em cidades de porte médio e em algumas capitais. Finalmente duas entrevistas ajudaram a completar a avaliação do programa.

Palavras-chave: Política habitacional; Paisagem urbana; Minha Casa Minha Vida. 


\section{INTRODUCTION}

Le programme Minha casa, minha vida ("Ma maison, ma vie"), le plus grand programme de construction de logements des trente dernières années, a profondément changé le paysage de nombreuses villes brésiliennes en y créant d'immense quartiers de maisons identiques, alignées au cordeau et très proches les unes des autres (figure 3). Lancé en 2009, au cours du second mandat de l'ancien président Luíz Inácio Lula da Silva, il est devenu l'un des principaux fleurons des politiques gouvernementales de Dilma Rousseff. Conçu pour stimuler la construction et à lutter contre les effets de la crise économique internationale, le programme est devenu un instrument pour réduire le déficit de logements dans le pays. Si elle est menée à bien, la troisième phase du programme, lancée en mars 2016, portant le total de maisons construites à 5,75 millions de maisons pour 23 millions de bénéficiaires, pourrait lui faire dépasser les résultats de la BNH (Banque nationale du logement), créée sous la dictature militaire (1964-1985).

Récemment, le programme a fait l'objet d'une controverse quand Michel Temer (PMDB) a pris ses fonctions en tant que président par intérim après la destitution de Dilma Rousseff. Les membres du MTST (Mouvement des travailleurs sans-abri) ont occupé le hall du Secrétariat de la Présidence, sur l'avenue Paulista de São Paulo, pour exiger que le nouveau ministre des Villes ne revienne sur sa décision de révoquer l'ordre du gouvernement Dilma Rousseff autorisant la construction de nouvelles unités.

Au moment où le programme semble menacé il est donc opportun d'essayer de faire le point. Sans prétention à faire une analyse approfondie, nous avons choisi un angle spécifique et, après une brève présentation de son histoire, nous avons cherché à repérer la marque du programme sur le paysage des villes brésiliennes sur des photos (prises sur le terrain ou récoltées sur Internet) et des images verticales de Google Earth, en particulier dans les villes de taille moyenne, mais aussi dans certaines capitales. Enfin deux interviews nous aideront compléter l'évaluation du programme, en en soulignant les forces et les faiblesses.

\section{LE PROGRAMME ET SON ÉVOLUTION}

La première étape du programme a atteint, au moins sur le papier, son objectif de fournir entre 2009 et 2011 un million de maisons à la population ayant un revenu allant jusqu'à dix salaires minimums (environ 1400 Euros - Reais/Euros : 3,5/1). Selon le rapport de la Cour des comptes (TCU) à la fin de 2010 un peu plus d'un million de contrats pour la construction ou le financement de logements avaient été signés, soit $100,4 \%$ de l'objectif du programme. Toutefois, sur ce total seulement 238000 (23\%) avaient été livrés. Au cours des deux premières phases du programme de logement ont été investi environ 240 milliards de Reais (68,6 milliards d'Euros).

Sur les deux millions de logements prévus pour 2014, 1,2 million étaient destinés aux familles gagnant jusqu'à 1600 Reais par mois (457 Euros). Pour les familles dont le revenu était inférieur à 3100 Reais (885 Euros), l'objectif était de construire 600000 logements et 200000 pour ceux qui gagnaient jusqu'à 5000 Reais (1 428 Euros). En outre, le gouvernement a répondu à la demande des sociétés de BTP et a augmenté la valeur moyenne des logements pour la population à faible revenu de 42000 à 55000 Reais $(12000$ à 15715 Euros.

En 2014, année où elle a été candidate à la réélection, la présidente avait promis que la troisième phase du programme de logements livrerait trois millions de logements. En mars 2016, au milieu de la procédure de destitution lancé contre elle et un jour après que le PMDB, son principal soutien en dehors du Parti des Travailleurs, ait rompu officiellement avec elle, a été lancée la troisième phase du programme, dont le but était de fournir deux millions de logements pour 2018 (et donc un total réduit d'un million). Selon le Ministère des Villes, la prévision du gouvernement était alors d'investir environ 210 milliards de Reais (60 milliards d'Euros) dans cette nouvelle étape. 
Toujours en mars 2016, le gouvernement fédéral a lancé le portail du programme (http://www. minhacasaminhavida.gov.br/) qui rassemble les informations sur le programme. Le 17 octobre 2016 il indiquait un total de 4219366 maisons déjà commandées, 294,5 milliards de Reais (80 milliards d'Euros) investis, 2632953 familles ayant reçu un logement, soit 10,5 millions de personnes relogées et 1586413 sur le point d'obtenir leurs maisons.

Si l'on cartographie les actions du programme (figure 1) on voit que de nouvelles maisons ont été effectivement construites dans tout le pays, en priorité dans les régions déficitaires. Cependant, il existe une différenciation des éléments qui composent ce déficit. En ce qui concerne les logements insalubres, le Maranhão est l'État qui a le pourcentage le plus élevé, suivi de près par le Pará, le Piauí, le Ceará et la Bahia. Mais la composante décohabitation représente plus d'un tiers des ménages dans le Pará, le Ceará, le Rio Grande do Norte, la Paraíba, le Sergipe, l'Alagoas, la Bahia, le Minas Gerais, Rio de Janeiro et le Rio Grande do Sul. Dans d'autres États c'est la charge excessive du loyer dans le budget de la famille qui a poussé les gens à chercher à bénéficier des actions du programme.

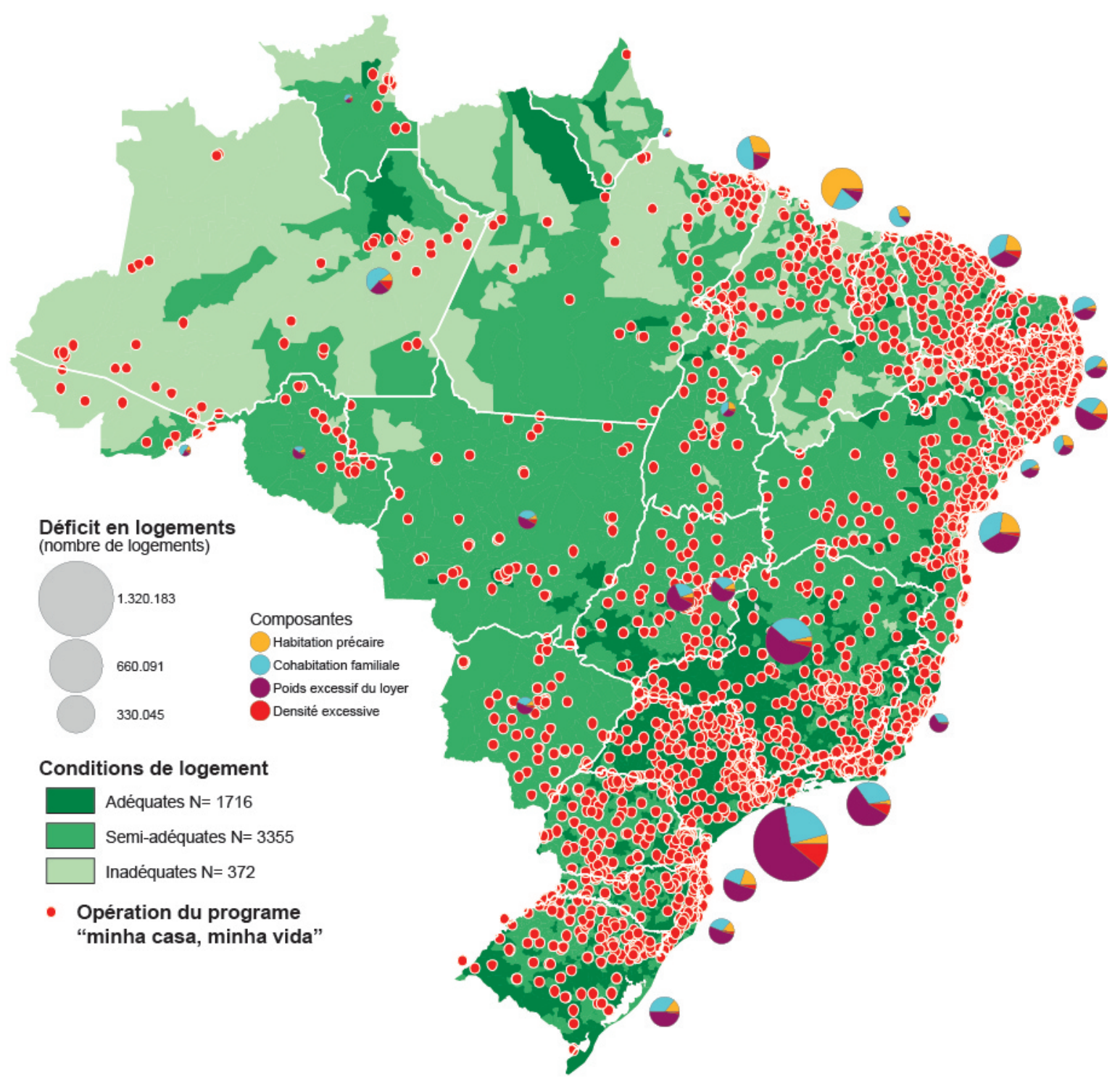

Figure 1 - Répartition des actions du programme

Source: Hervé Théry et Neli Aparecida de Mello-Théry, Atlas do Brasil, Edusp, $3^{\mathrm{a}}$ edição sous presse. 
THERY, H.

Tableau 1 - Tranches de revenus et valeurs des maisons, nouvelles valeurs

\begin{tabular}{c|c|c|c|c|c} 
Tranches & $\begin{array}{c}\text { Nouvelles tranches } \\
\text { de revenu }\end{array}$ & Anciennes tranches & $\begin{array}{c}\text { Novos valores des } \\
\text { maisons }\end{array}$ & Subvention & $\begin{array}{c}\text { Anciennes valeurs des } \\
\text { maisons }\end{array}$ \\
\hline Tranche 1 & 1800 & 1600 & 96000 & 86400 & 76000 \\
\hline Tranche 1,5 & 2350 & & 135000 & 45000 & \\
\hline Tranche 2 & 3600 & 3275 & 225000 & 27500 & 190000 \\
\hline Tranche 3 & & 5000 & 225000 & 0 & 190000
\end{tabular}

Source: Ministério das Cidades

\section{PAYSAGES URBAINS}

Les images qui suivent montrent les effets du programme sur les paysages urbains, vus sur le terrain ou sur internet, que ce soit des photos de l'inauguration des nouveaux ensembles ou des vues verticales obtenues à partir de Google Earth. La figure 2 situe les villes mentionnées.

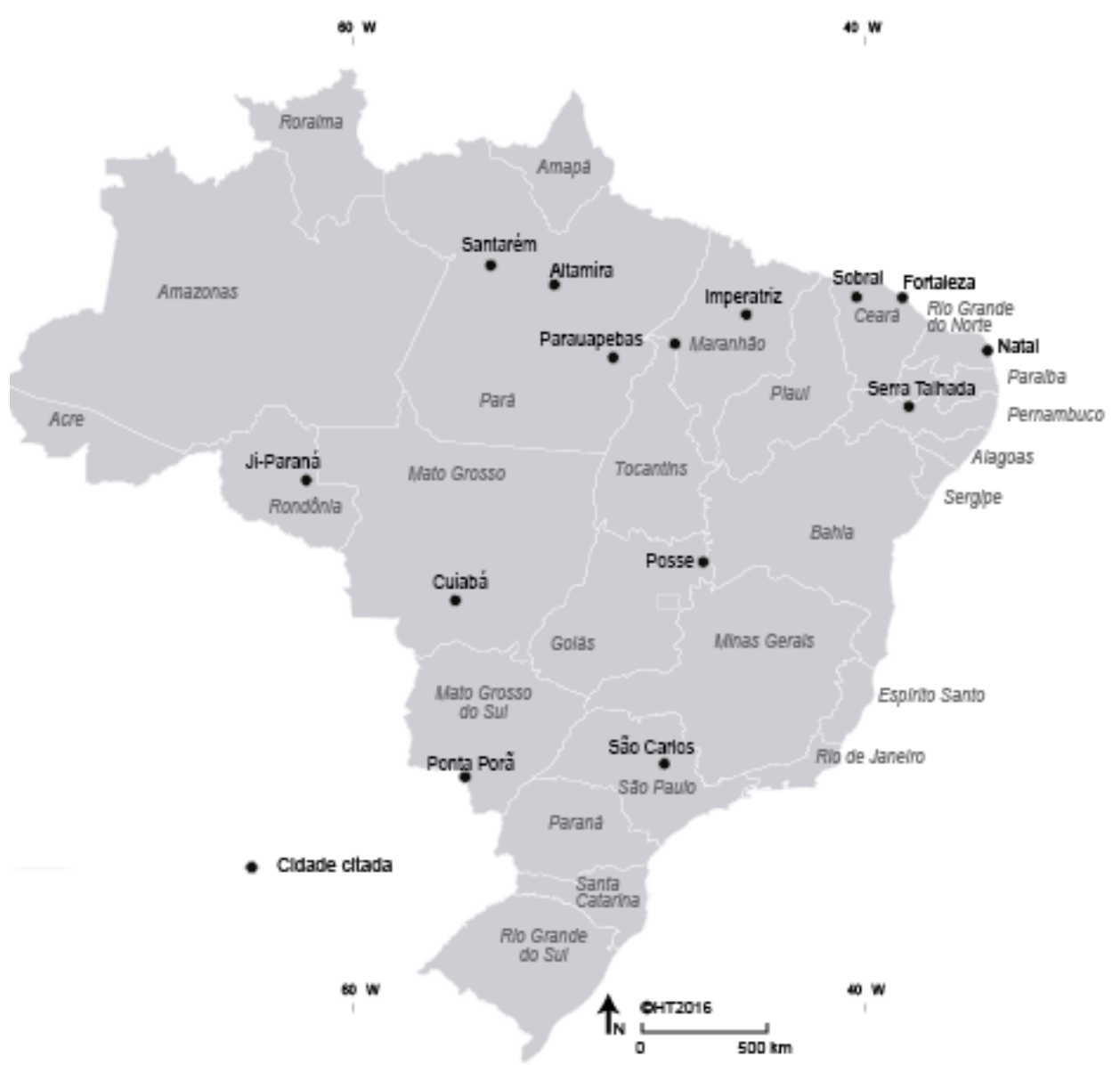

Figure 2 - Villes citées

Ce qui est frappant sur ces photos est l'uniformité des paysages produits, en raison de la normalisation des maisons. Dans le cas de Serra Talhada (photo 1 sur la figure 3), ville de Pernambuco située à $450 \mathrm{~km}$ de Recif), le Conjunto Habitacional Poço da Cruz a été créé, au km 418 de la route BR232, près du centre de Serra Talhada. Sa planification avait été prévue en deux étapes, la seconde formée par Poço da Cruz III (195 maisons), et le Poço da Cruz IV (500 maisons, livrées le 1er février 2016). Avec la livraison de ces 500 logements ce sont environ 1200 familles qui s'ajoutent au quartier de Vila Bela, et en font un des plus peuplés de la ville. Les maisons de Poço da Cruz IV ont une superficie $32,23 \mathrm{~m} 2$, divisée en deux chambres, couloir, salon, salle de bains, cuisine et buanderie, un modèle qui se répète dans d'autres ensembles du programme. 


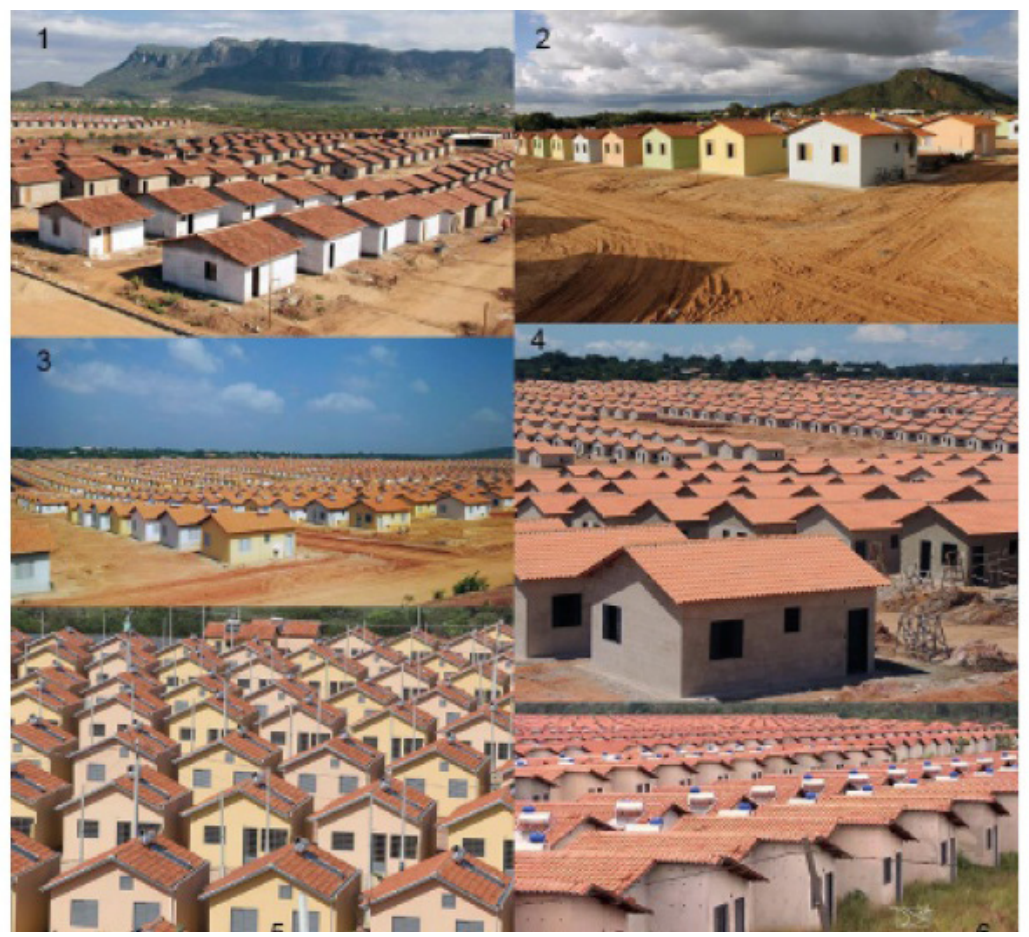

Figure 3 Paysages du programme: 1) Serra Talhada (PE), 2) Posse (GO), 3) Jí-Paraná (RO), 4) Santarém (PA), 5) São Carlos (SP), 6) Imperatiz (PA).

Sources: 1) Serra Talhada (PE), http://www1.duarteconstrucoes.com.br/cms/opencms/duarte2011/pt/noticias/ arquivos/0031.html; 2) Posse (GO), http://www.dinomarmiranda.com/2015/05/prefeitura-de-posse-go-vai-realizardia.html; 3) Jí-Paraná (RO), http://www.portaljipa.com.br/?pg=noticias\&i=MTY0MzU=; 4) Santarém (PA), http:// www.brasil.gov.br/governo/2016/05/residencial-salvacao-transforma-vidas-em-santarem-no-para; 5) São Carlos (SP), https:/wordpaulotamer.wordpress.com/2015/10/14/presidente-dilma-roussef-e-governador-geraldo-alckminparceiros-no-programa-minha-casa-minha-vida/; 6) Imperatiz (PA), http://g1.globo.com/ma/maranhao/bom-diamirante/videos/v/construcao-do-minha-casa-minha-vida-segue-atrasada-em-imperatriz-ma/4855899/.

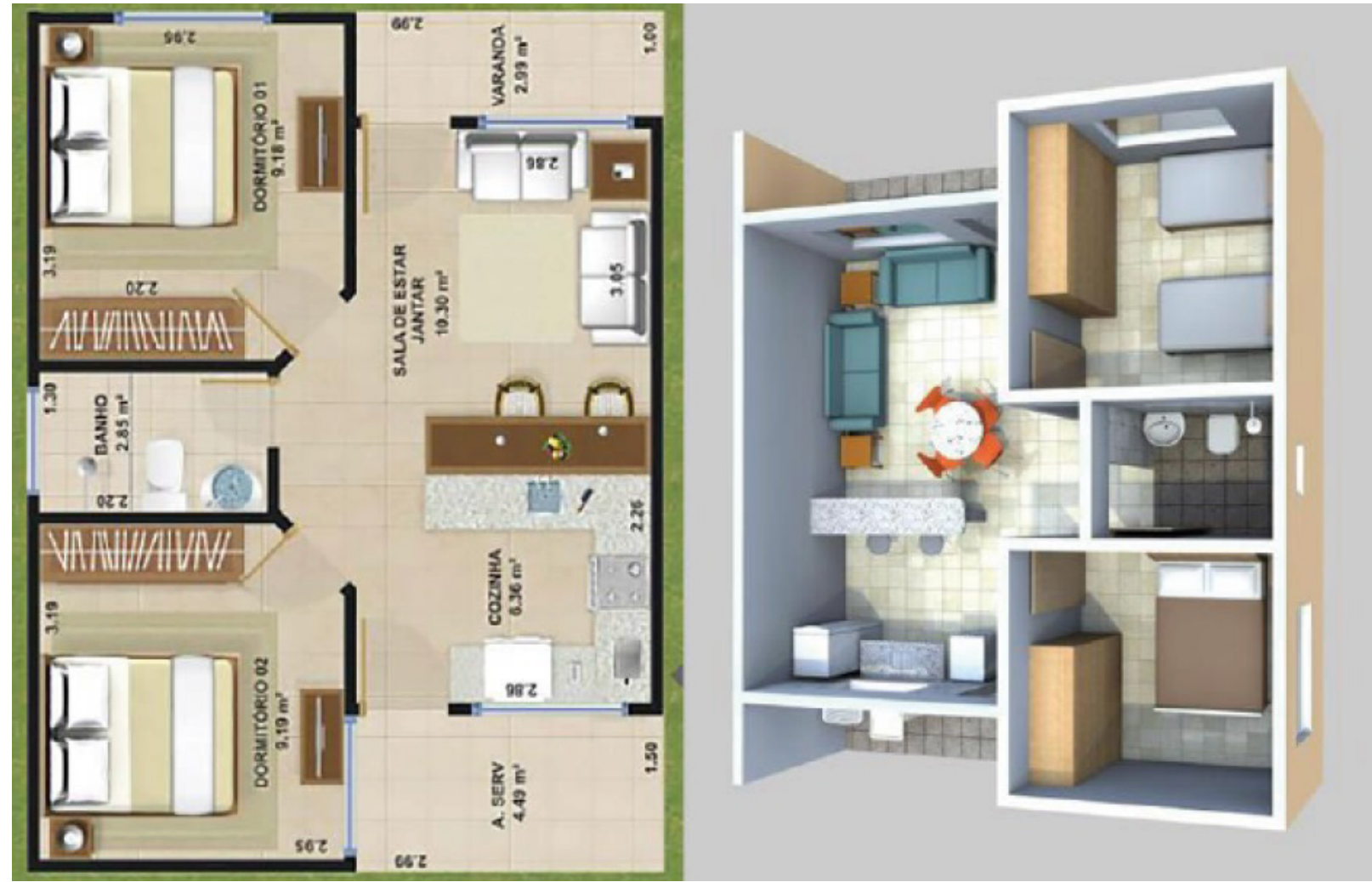

Figure 4 - Plan typique des maisons du programme

Source: http://www.cantodasideias.com/plantas-de-casas-populares/ 


\section{VILLES MOYENNES}

Dans les villes moyennes l'impact du programme est fort parce qu'il a conduit à la construction de quartiers qui deviennent souvent les plus grands de la ville. L'option « images historiques » Google Earth permet de suivre les étapes de leur apparition à la périphérie des villes, comme à Ponta Pora et Sobral, sur la figure 5 et dans les paragraphes suivants.
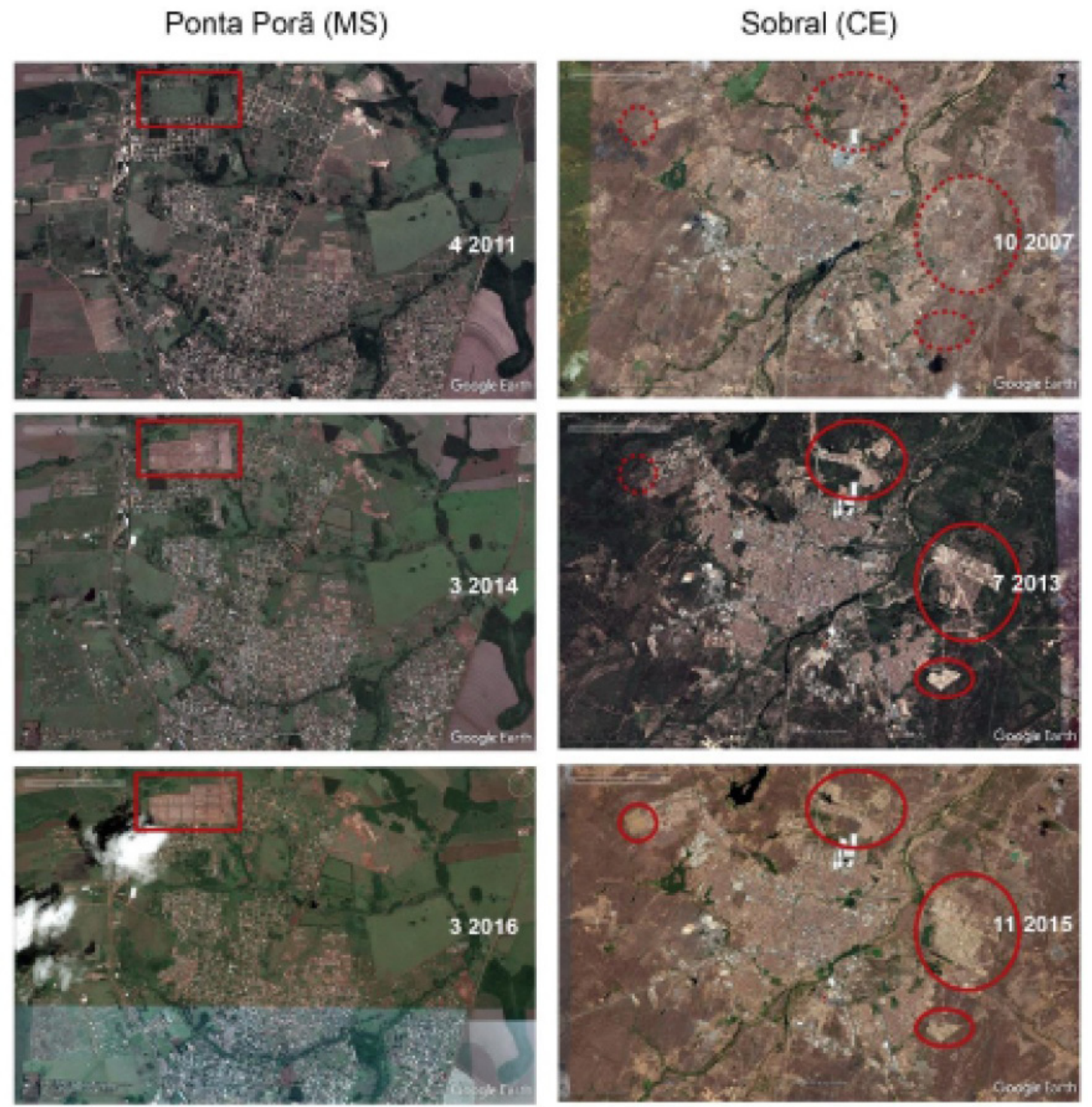

Figure 5 - Nouveaux quartiers dans deux villes moyennes Source: Google Earth

\section{PONTA POR ̃}

Le programme a livré en juillet 2016 plus d'un millier de logement dans la ville de Ponta Porã (Mato Grosso do Sul, $330 \mathrm{~km}$ de Campo Grande), le plus grand ensemble de logements jamais construit dans l'État, et l'un des plus importants au Brésil. Sa construction avait commencé en 2014, le financement fédéral étent complété par le gouvernement de l'État et les municipalités pour 10\% des montants (donation de terrains). 
La cité, qui a reçu le nom de Kemel Saad, est située dans le quartier Jardim dos Eucaliptos. Au total, y ont été investis environ 63,7 millions de Reais (18,2 millions d'Euros) dont 3 (850 000 Euros) par le gouvernement de l'État. Les maisons, construites sur 250 mètres carrés de terrain, ont 41,6 $\mathrm{m}^{2}$ et disposent de deux chambres à coucher, salon, cuisine et salle de bains. Parmi ces logements, 31 sont adaptés pour les personnes handicapées et 832 sont conçus pour les femmes. Les murs de la cuisine, salle de bains et buanderie, où a été installé le lavoir, sont carrelés sur une hauteur d'un mètre et demi, toutes les portes intérieures sont en bois, et celles qui donnent accès à l'extérieur sont en fer.

\section{SOBRAL}

En mars 2016, ont été livrés des maisons du programme à 976 familles de Sobral (Ceará, à 240 km de Fortaleza). Le même jour, 1458 autres familles ont reçu un logement au cours de cérémonies simultanées dans quatre autres États: Rio Grande do Sul, Mato Grosso do Sul, São Paulo et Minas Gerais, au total 2434 familles. En 20152911 logements avaient été livrés dans le Ceará, 768 à Fortaleza et 2143 dans l'intérieur. Le total des investissements a été de 229 millions de Reais (65,4 millions d'Euros), dont une contribution du gouvernement de l'État de 11 millions de Reais (3,14 millions d'Euros).

À Sobral, les maisons font partie du quartier résidentiel « Fierté Tropicale ( Orgulho Tropical), trois modules qui, ensemble, comptent 3368 maisons pour un investissement de 198,5 millions de Reais (56,7 millions d'Euros), dont 10,11 (2,9) de l'État. Chaque maison est de 42,9 mètres carrés, divisés en salle de séjour, cuisine, deux chambres, salle de bains et buanderie.

Tableau 2 - Maisons du programme livrées à Sobral

\begin{tabular}{c|c|c} 
Module & Unités & Investissements (millions de R\$) \\
\hline Orgueil Tropical 1 & 1252 & 73,86 \\
\hline Orgueil Tropical 2 & 832 & 49,08 \\
\hline Orgueil Tropical 3 & 1280 & 75,52 \\
\hline Total & 3364 & 198,48
\end{tabular}

\section{PARAUAPEBAS}

À Parauapebas le programme a contribué de manière significative à l'expansion de la zone urbaine, qui a doublé entre 2006 et 2001, une croissance portée à la fois par l'effet du programme et par la situation financière favorable de la municipalité, qui bénéficie des royalties provenant de l'exploitation des mines de fer de Carajás. 

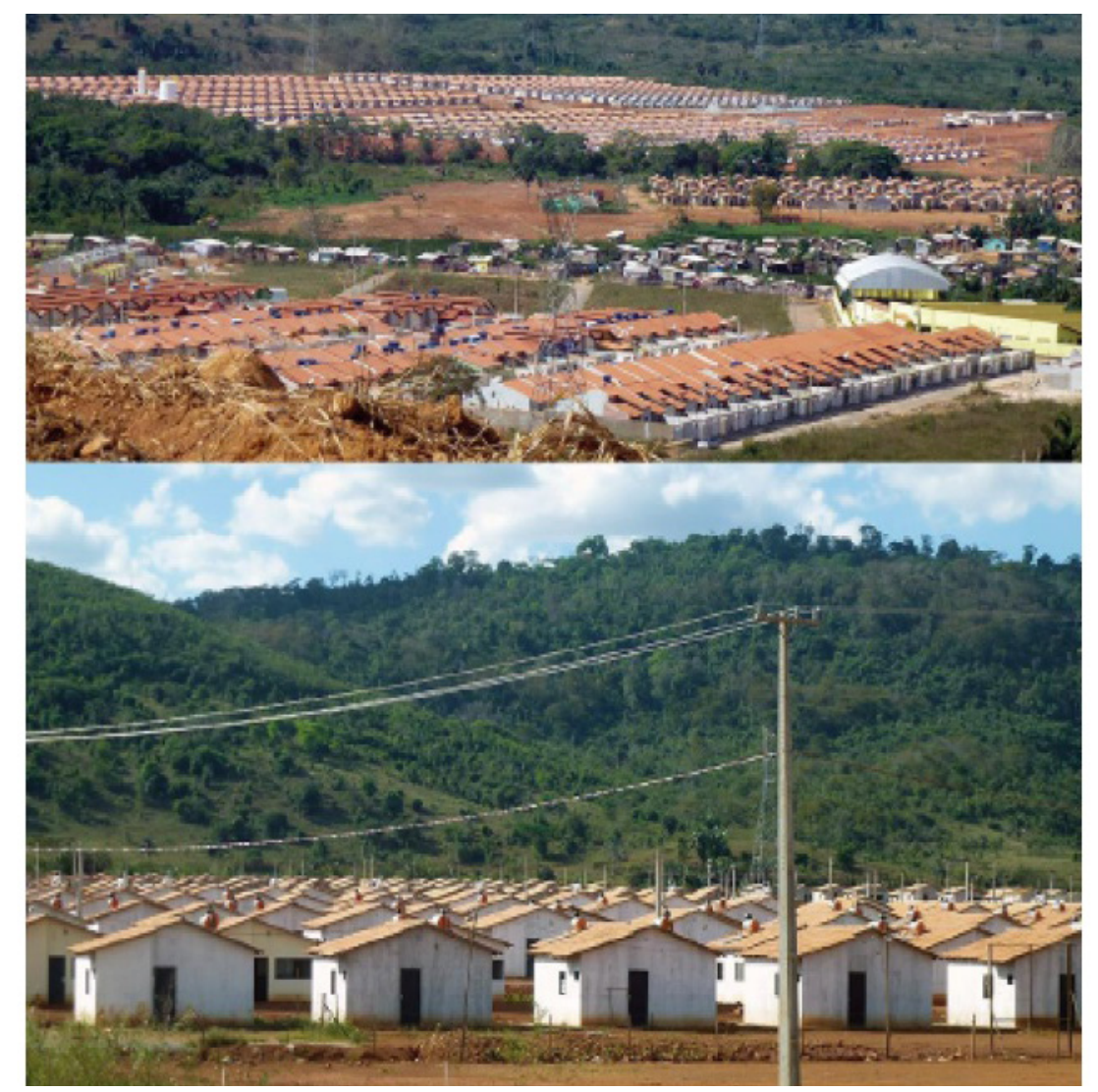

Figure 6 - Maisons du programme à Parauapebas (PA) Photos Hervé Théry 2009

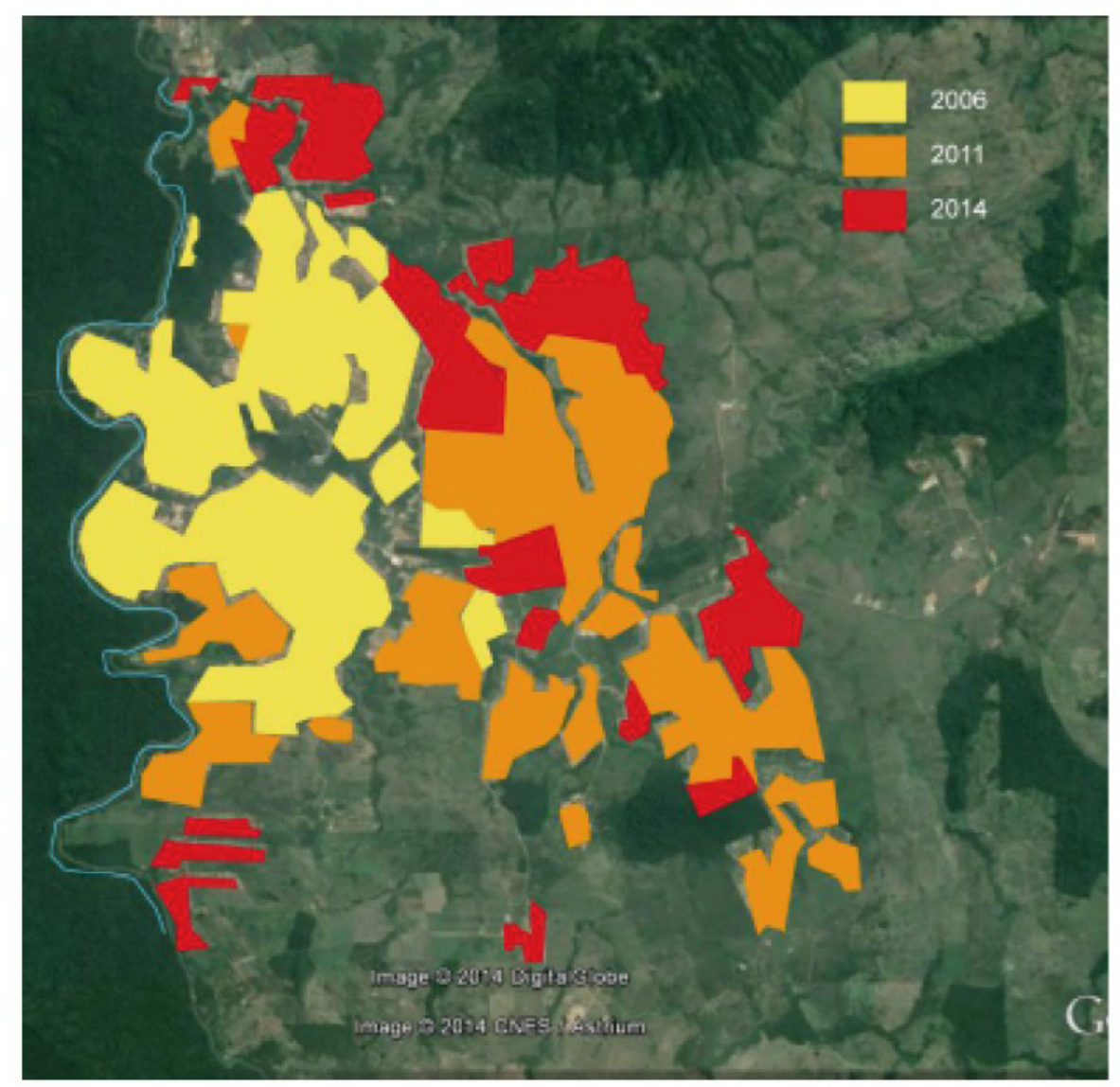

Figure 7 - Parauapebas 2006-2013 Source: Google Earth e Hervé Théry 


\section{EFFETS SUR LE PAYSAGE DES CAPITALES}

L'effet du programme est si fort qu'il est sensible même à la périphérie de certaines capitales d'États. Lorsque l'on voyage en avion les nouveaux quartiers sont clairement visibles dans le paysage, car ils sont généralement construits dans des zones jusque-là non urbanisées. Le remplacement du le vert de la végétation par le rouge vif de la terre nue, et la géométrie rigide des alignements de centaines de maisons saute aux yeux des voyageurs.

\section{DE NOUVEAUX QUARTIERS GÉOMÉTRIQUES}

Le cas de Fortaleza est exemplaire car la capitale du Ceará aura, quand il sera terminé, le plus grand projet Minha Casa Minha Vida du pays, dans le quartier d'Ancuri.

Plus de 1700 familles y bénéficieront de la construction de la première étape du résidentiel Luiz Gonzaga. Lancé en octobre 2015, il abritera 1760 maisons et recevra des investissements de 130,6 millions Reais (37,3 millions d'Euros) de l'Union et de l'État. En tout, le programme compte à Fortaleza 25088 logements répartis sur 29 sites. Les logements sont de 47,3 $\mathrm{m}^{2}$ divisé en deux chambres, cuisine, salle de bains, salon et véranda.

C'est également le premier ensemble, au Ceará, de la modalité dans lequel des organisations non gouvernementales, comme des associations et des coopératives, présentent leurs demandes et sont responsables de la gestion du chantier. Dans ce cas, les familles, dont le revenu brut mensuel ne doit pas dépasser 1600 Reais (457 Euros), commencent à payer, après la mise à disposition du logement, un maximum de $5 \%$ de leur revenu familial.

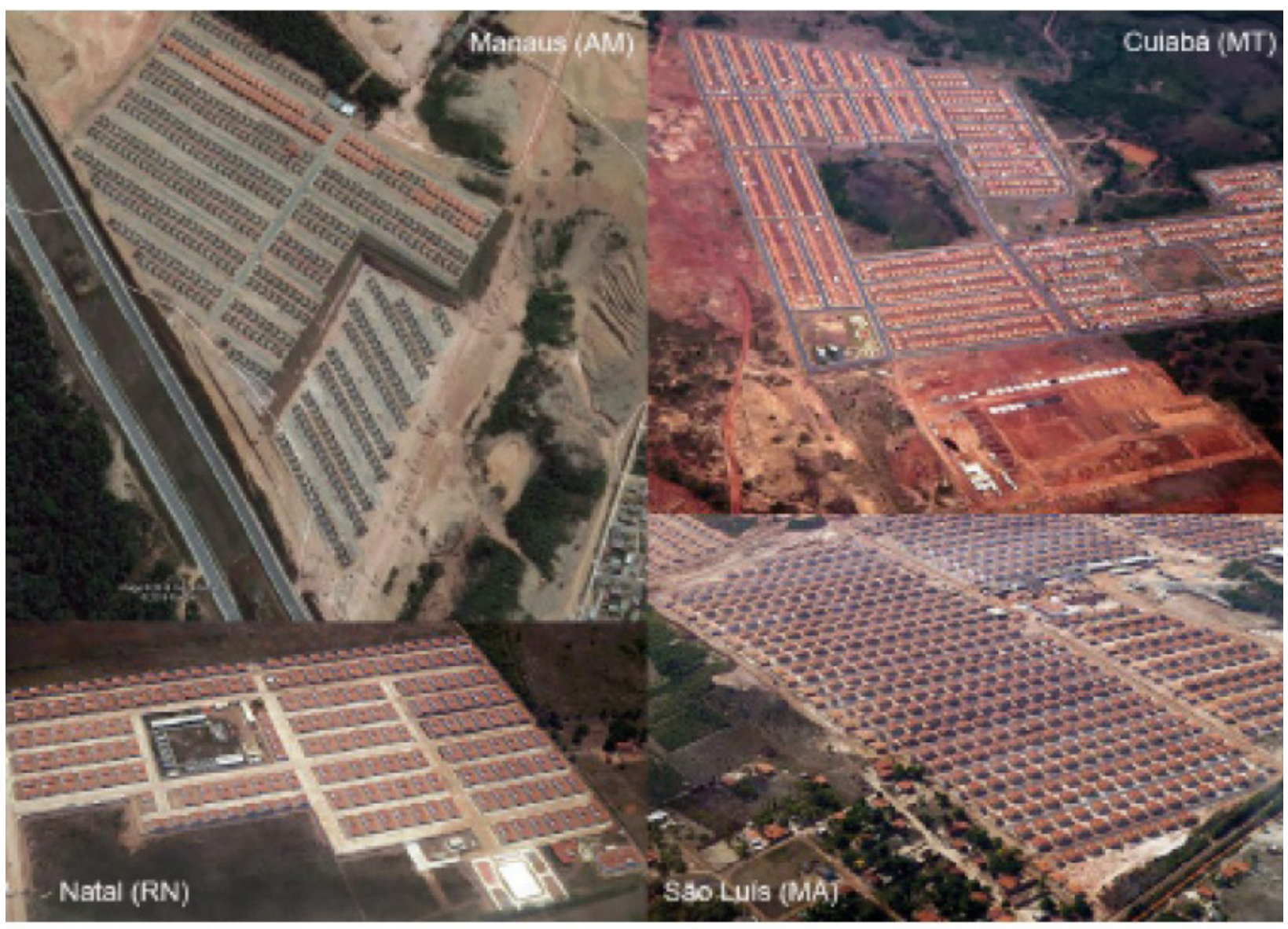

Figure 8 - Vues aériennes de nouveaux quartiers

Source: Photos Manaus Google Earth, Cuiabá, Natal e São Luís Hervé Théry, 2015 e 2016 
2162014

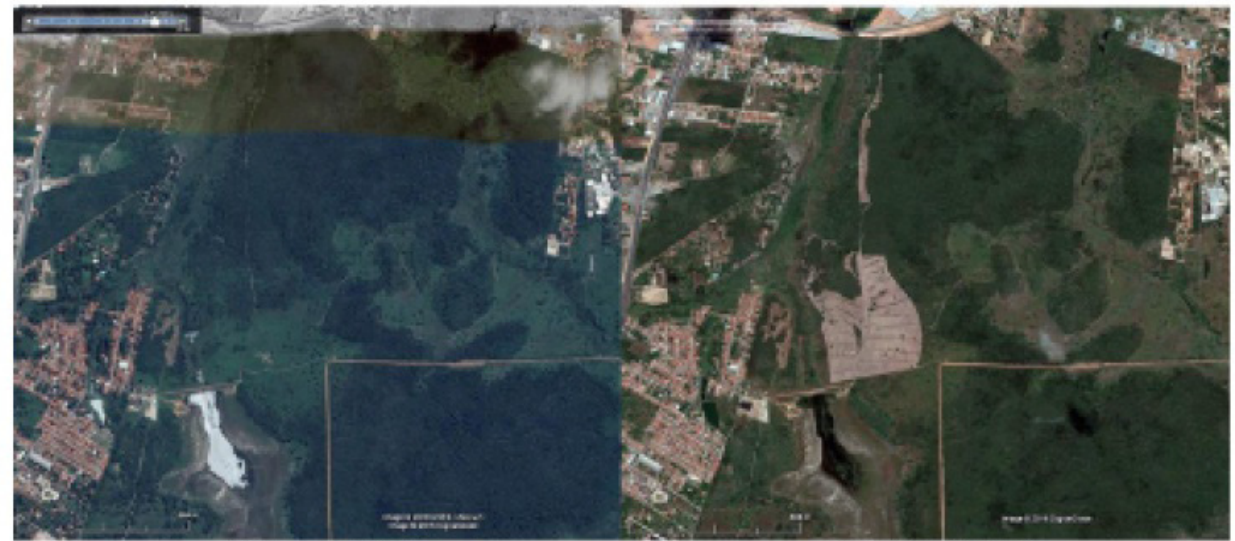

3172015

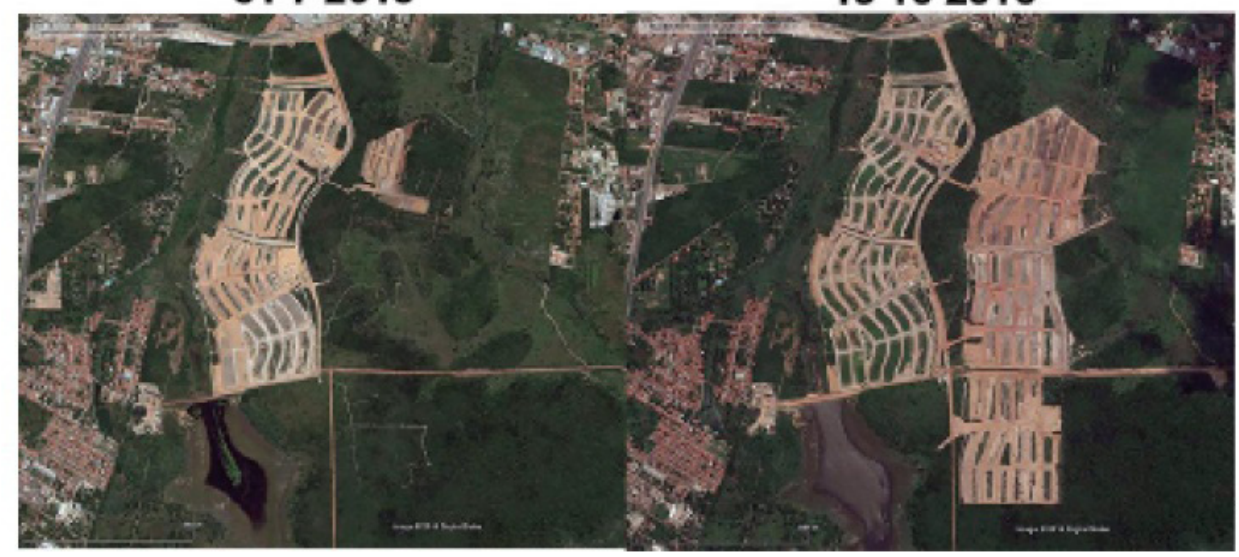

Figure 9 - Étapes de la construction de la cité Luis Gonzaga

Source: Google Earth

La demande de logements est si forte que l'attribution est faite par tirage au sort, après établissement des priorités pour certaines catégories de bénéficiaires. Le premier tirage au sort était réservé aux familles inscrites jusqu'au 13 novembre 2014, 88068 d'entre elles avaient été enregistrées et ont pu participer cette fois. Sur un total de 3304 logements ont d'abord été tirés au sort 3\% destinés uniquement aux familles comptant un ou des handicapés, et 3\% pour les familles dont le chef ou le conjoint est âgé de plus de 60 ans.

Les autres familles inscrites pourront être incluses dans d'autres occasions, le nombre de maisons des prochains tirages variant en fonction du degré d'avancement des travaux. Six critères prioritaires ont été retenus: les familles vivant dans les zones à risque; celles qui sont dirigés par des femmes; celles qui ont des membres âgés ou handicapés; celles qui sont bénéficiaires de loyers sociaux; les familles nombreuses (plus de trois personnes partageant la même chambre); et celles qui comptent des personnes atteintes de maladies chroniques graves comme le cancer, le sida, les maladies cardiaques ou l'hémophilie.

Une tranche de $75 \%$ des parts sera affectée familles qui répondent à cinq des six critères, les autres $25 \%$ à celles qui répondent à quatre critères. Pour chaque groupe, $30 \%$ de plus seront sélectionnés en liste supplémentaire. Les gagnants recevront les maisons après validation des informations fournies lors de l'inscription, si elles sont fausses, la personne sélectionnée sera exclue du processus.

$\mathrm{Au}$ total, les impacts du programme sont assez forts pour remodeler la périphérie de grandes villes, comme Cuiabá et São Luis, qui ont reçu en quelques années bon nombre de nouveaux quartiers (figure 10). 
Cuiabá (MT)
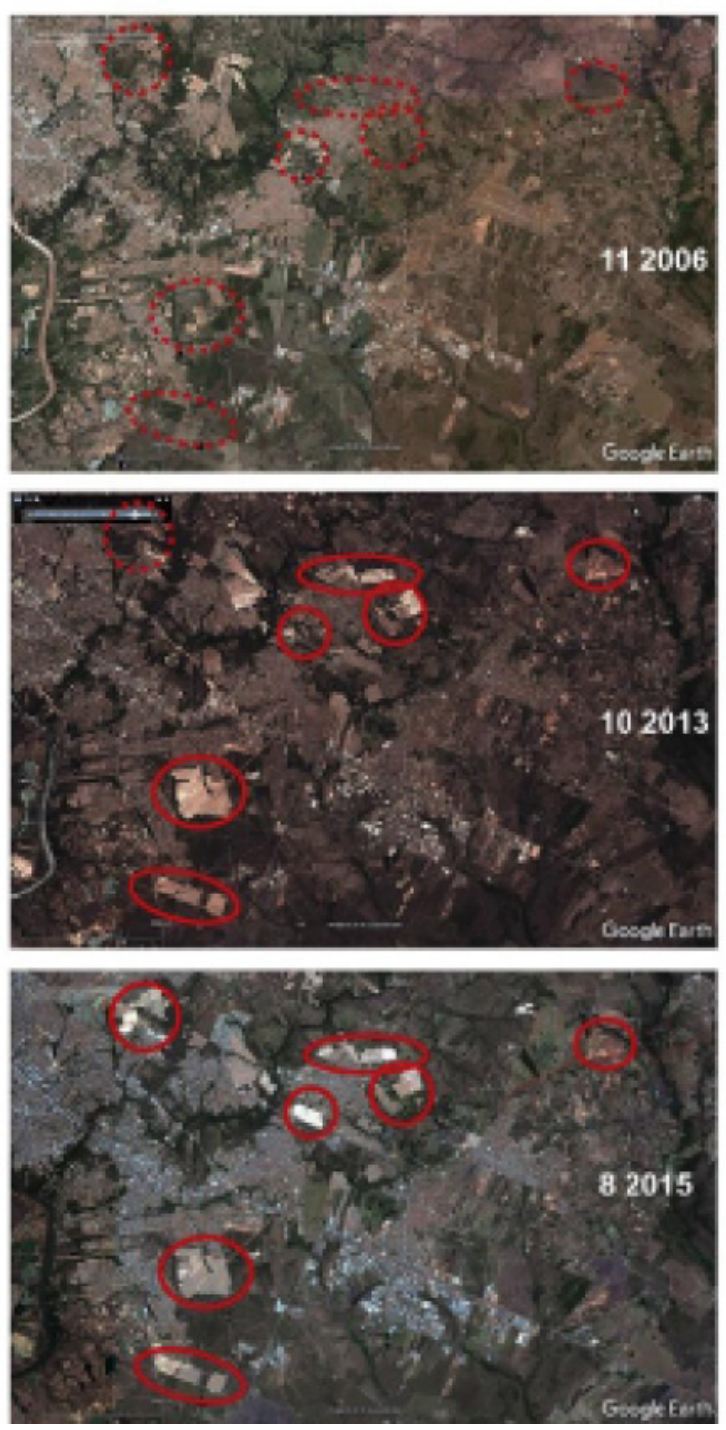

Săo Luis do Maranhăo (MA)
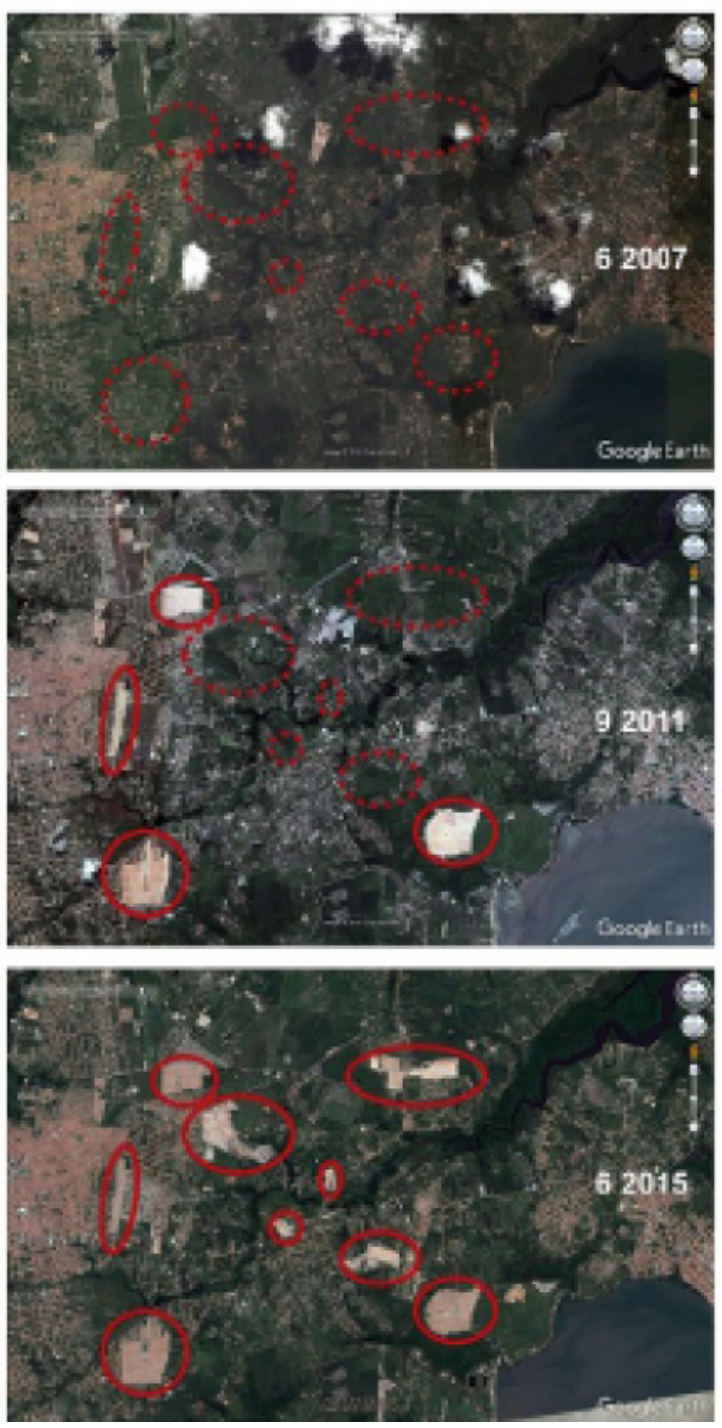

Figure 10 - Étapes de la construction de nouveaux quartiers Source: Google Earth et Hervé Théry

\section{CONSIDERATIONS FINALES}

Enfin, deux interviews donnent des visions contrastées du programme. Le premier est celui d'Adauto Cardoso, professeur à l'Université fédérale de Rio de Janeiro et chercheur à l'Observatoire des Métropoles, qui suit la question du logement et notamment les actions de Minha Casa Minha Vida. Dans cette interview (publiée sur le site de l'Observatoire des Métropoles), il commente les principaux problèmes de la première étape du programme et leurs effets sur la configuration des régions métropolitaines du Brésil.

La deuxième est celle d'Inês Magalhães, sociologue diplômée de l'Université Catholique de São Paulo (PUC-SP) et spécialiste de la planification et de la gestion. Depuis 2003, elle a travaillé au Ministère des villes, d'abord en tant que directrice, puis en tant que secrétaire nationale au logement et, enfin en tant que ministre (pendant 21 jours, la veille de la mise en accusation de Dilma Rousseff). Dans l'interview (publiée sur le site Web du magazine Forum), il fait le point sur le programme au moment de la transition politique entre les gouvernements Rousseff et Temer. 


\section{ADAUTO CARDOSO}

D'après les études de l'Observatoire des Métropoles, quels problèmes ont été rencontrés dans la première étape de Minha Casa, Minha Vida?

Nous pouvons parler de quatre problèmes majeurs dans le programme fédéral. Le premier, qui apparaît dans toutes les analyses que nous avons faites, est lié à l'emplacement des nouvelles cités, puisque la plupart d'entre elles sont construites dans des zones périphériques, peu en rapport avec le tissu urbain. Nous avons des exemples de maisons complètement isolées par rapport à la ville, ce qui a des implications en termes d'infrastructures de transport et en termes de la qualité de vie pour les populations qui y vivent $[\ldots]$.

En outre le programme ne réussit pas à prendre en charge la question foncière, le problème de la terre et de la valeur de celle-ci [...]. Ainsi, plus on investit dans le programme, plus les terres prennent de valeur, plus le coût de l'unité va augmenter, et plus de cet argent sera capturé par la propriété de la terre, par les propriétaires. Le résultat est que vous utilisez des ressources publiques, vous accordez des subventions pour aider la population à faible revenu, mais cela bénéficie indirectement aux propriétaires de la terre $[\ldots]$.

Et quels sont les autres problèmes?

Un deuxième défaut vient des technologies utilisées dans ces entreprises. Nous avons observé un certain nombre de problèmes à cet égard, puisque les plans sont des solutions conventionnelles et peu adaptables à la population à faible revenu [...]. Par exemple, les projets ont été réalisés avec des coûts de charges relativement élevés, ce qui créer de difficultés pour les familles à faible revenu. [...]. Un autre problème [vient des] solutions constructives [...] Par exemple, certains constructeurs utilisent un « moule tunnel » qui produit des murs en béton, si le résident veut percer un trou pour placer un tableau ou ouvrir une porte, il ne le peut pas. [...] Les solutions architecturales sont classiques et sont répétées à travers le Brésil, sans une adaptation aux besoins régionaux de la population [...].

D'autres difficultés viennent du modèle de copropriété fermée, conçu pour le mode de vie de la classe moyenne, peu adaptée à ces nouveaux développements [...]. Dans le cas des projets construits dans des zones très reculées se pose toujours le problème de l'absence de services pour la population, car les zones commerciales ne sont pas autorisés dans ces ensembles purement résidentiels.

Il y a des résultats différents entre les États?

Nous avons constaté une claire distorsion régionale dans la répartition des ressources. [Dans la première phase] Bahia a reçu 11,6\% des contrats depuis 2011, et São Paulo 19\% . D'autre part, il y a des États qui ont reçu peu de ressources, comme le Ceará (2,2\%), et l'Espírito Santo (1,8\%). Le Pará, par exemple, a reçu moins de ressources que l'État d'Alagoas, alors qu'il souffre d'une grave pénurie de logements.

Et si l'on regarde la dynamique de la distribution au sein de chaque État nous voyons aussi la même distorsion, dans le cas de Rio de Janeiro la plupart des ressources ont été allouées à la région métropolitaine, et pas à toutes les municipalités. Itaboraí, par exemple, qui a une très forte dynamique urbaine et une très grande demande de logements pour les populations à faible revenu, n'a reçu aucune cité pour la tranche de zéro à trois fois le salaire minimum, c'est la capitale qui a reçu la quasi-totalité des fonds pour ces tranches de revenu. Et en dehors de la région métropolitaine seulement deux municipalités ont reçu des fonds: Volta Redonda et Cabo Frio. 


\section{INÊS MAGALHÃES}

Vous qui étiez au ministère des Villes depuis le son premier titulaire, Olivio Dutra (PT), quelle évaluation pouvez-vous faire depuis la première unité livrée jusqu'à ce jour? Quel est le résultat concret dans la vie des gens?

Lorsque nous avons lancé le Minha Casa, Minha Vida (MCMV), en 2009, nous avions de très grands défis à relever. Parmi eux, répondre à demande de la population à faible revenu et combler le déficit de logements dans le pays. Aujourd'hui, sept ans plus tard, nous pouvons dire que nous sommes sur la bonne voie pour surmonter ces défis, plus de $90 \%$ de nos ressources ont été allouées à des familles dont le revenu est inférieur à trois fois le salaire minimum.

Depuis le lancement des contrats ont été signés pour la construction de 4,25 millions de logements. À la fin de la troisième phase de MCMV, 5,75 millions d'unités bénéficieront toucheront 23 millions de personnes. Aujourd'hui, plus de 10 millions de personnes vivent dans une maison du programme et le MCMV est présent dans plus de 5300 municipalités . Cela signifie que les familles à faible revenu vivant dans plus de $96 \%$ des municipalités brésiliennes ont accès à ce programme de logement [...].

Quel est le calendrier établi par le gouvernement fédéral pour le Minha Casa, Minha Vida d'ici la fin de cette année?

En mars de cette année, le gouvernement a annoncé la troisième phase du programme avec l'objectif de deux millions d'unités dans la période 2015-2018. Pour 2016, l'objectif est de 480000 unités, dont 110000 pour la tranche 1 (familles ayant un revenu jusqu'à 1,800 Reais / 514 Euros).

Que manque-t-il pour rendre le droit au logement atteigne un plus grand nombre de bénéficiaires?

A la fin de MCMV 3, auront été passés des contrats pour 5,75 millions d'unités. Quand elles auront été livrées, il y aura plus de 23 millions de bénéficiaires, c'est-à-dire qu'une personne sur huit dans le pays sera bénéficiaire du programme. Je souligne que la plupart des avantages pour la tranche $1(69 \%)$ ont été alloués à des familles dont le revenu ne dépasse pas 800 Reais (229 Euros) par mois, ce qui montre bien l'adéquation entre le programme et les caractéristiques du déficit de logements existants dans le pays.

\section{REFERENCES BIBLIOGRAPHIQUES}

BORGES, Lucio. Minha Casa Minha Vida entrega hoje maior conjunto habitacional de MS em Ponta Porã, 20 de julho de 2016, http://paginabrazil.com/minha-casa-minha-vida-entrega-hoje-maior-conjunto-habitacional-de-ponta-pora/

Duarte irá construir mais 695 casas populares em Serra Talhada, 31/01/2012, http://www1.duarteconstrucoes. com.br/cms/opencms/duarte2011/pt/noticias/arquivos/0031.html

Fortaleza inicia maior obra do Minha Casa Minha Vida do País. 17/10/2015, http:/www.opovo.com.br/app/ opovo/cotidiano/2015/10/17/noticiasjornalcotidiano,3520330/fortaleza-inicia-maior-obra-do-minha-casa-minha-vida-do-pais.shtml.

Fortaleza terá maior projeto Minha Casa Minha Vida do país, 9/10/2015, http://www.vermelho.org.br/ noticia/271631-1

JANUÁRIO, Wilame. Minha Casa, Minha Vida: Sobral recebe maior empreendimento do interior do Ceará, 07/3/2016, http://www.ceara.gov.br/sala-de-imprensa/noticias/15877-minha-casa-minha-vida-sobral-recebe-maior-empreendimento-do-interior-do-ceara 
MATOSO, Filipe. Governo lança terceira fase do programa Minha Casa, Minha Vida, 30/03/2016, http:/g1.globo.com/politica/noticia/2016/03/governo-lanca-terceira-fase-do-programa-minha-casa-minha-vida.html

Observatório das Metrópoles, Desafios para a política habitacional na $2^{\mathrm{a}}$ etapa do pro-grama minha-casa minha vida, sem data, http://observatoriodasmetropoles.net/index.php?option=com_content\&view=article $\& \mathrm{id}=1695 \% 3$ Adesafios-para-a-politica-habitacional-2o-etapa-do-programa-minha-casa\%20minhavida\&ca tid $=43 \% 3$ Anoticias\&Itemid $=114 \&$ lang $=\mathrm{pt}$

PIVA, Marco. Minha Casa, Minha Vida em risco?, 13/5/2016, http://www.revistaforum.com.br/2016/05/13/ minha-casa-minha-vida-em-risco/

RAMAlhoso, Wellington. Minha Casa, Minha Vida deu certo? Veja pontos positivos e negativos, 19/06/2016, http://noticias.uol.com.br/cotidiano/ultimas-noticias/2016/06/19/minha-casa-minha-vida-deu-certo-veja-pontos-positivos-e-negativos.htm

VIANA, Murilo. Prefeitura prevê entrega de 10 mil casas em 2016, 01/12/2015, http://diariodonordeste. verdesmares.com.br/cadernos/negocios/prefeitura-preve-entrega-de-10-mil-casas-em-2016-1.1445034 